\title{
Introduction
}

\section{Minisymposium on quality in medicine}

\author{
C.C. Huttin and M. Nabialczyk-Chalupowski \\ E-mails: chxyinfor@endepusresearchinc.com,christine.huttin@univ.amu.fr,mdphdjd@gmail.com
}

An initiative from the Polish Academy of Sciences in Paris (PAN), with participation of FNEGE, was launched in April 2018 to develop a consensus for quality indicators in medicine and to address challenges of health care systems, especially in Poland. A Polish report is already available with international and Polish experts' contributions, invited to this roundtable (www.medyk.com.pl).

This minisymposium provides complementary research ideas on ways to foster changes for quality improvements and covers some implementation issues.

The first paper by Professor E. Minvielle analyzes the complexity of health care delivery systems, from an organizational perspective, and underlines the priority to re design the systems incorporating more patient preferences.

The second paper by Dr Brosztek and Dr Domagała describes the way a survey instrument administered to the main stakeholders, at different levels of the national system, can provide a rapid feedback to better informed decision makers in Poland.

The third paper by Professor C. Huttin discusses the role of Patient Reported Outcomes in improving quality of care. Her experiences on pharmaceutical indicators in primary care (e.g. scoring methods on items representing trust, access to care, quality of information) may be relevant in relation to other initiatives such as Promis (US) or OECD PaRis on chronic care. 